\title{
Single vascular access for concomitant percutaneous coronary intervention and left ventricular assistance with Impella
}

\author{
Flávio Lyra, Breno O. Almeida, José Mariani Jr, Pedro A. Lemos, Adriano Caixeta \\ Department of Interventional Cardiology, Hospital Israelita Albert Einstein, São Paulo, Brazil
}

Adv Interv Cardiol 2021; 17, 2 (64): 218-222

DOI: https://doi.org/10.5114/aic.2021.107504

\section{Introduction}

Use of the Impella CP left ventricular assist device (LVAD) (Abiomed, Danvers, Massachusetts) requires a 14 French arterial introducer for its insertion, often performed via femoral access. An additional vascular access site is commonly used for coronary angiography or percutaneous coronary intervention $(\mathrm{PCl})$ - in general the contralateral femoral access. The need for multiple vascular access sites requires a longer procedure time and results in an increased risk of vascular complications. Additionally, many patients have associated peripheral vascular disease and therefore limited vascular access. Due to the large diameter of the Impella CP device sheath and its frequent use in high-risk $\mathrm{PCl}$ (e.g., complex unprotected left main coronary disease, multivessel disease, cardiogenic shock, advanced left ventricular dysfunction), reducing vascular complications by avoiding a second access is of paramount importance. The shaft diameter of the Impella CP catheter is 9 French, lower than the 14 French of its sheath. As previously reported, there is a novel safe alternative for the placement of an additional sheath up to 7F (1-4) using this available space between the inner diameter of the sheath and the Impella shaft, thereby avoiding the risks of an additional vascular access.

In this report we describe 3 cases in which the Single-access for High-risk PCI (SHiP) technique and Impella were used.

\section{SHiP technique description}

The SHiP has been described elsewhere $[1,2]$. In summary, the 14 French Impella sheath is introduced under a 0.035 " guidewire following the usual device im- plant technique. The Impella catheter is advanced from the femoral artery, through the aorta, and positioned in the left ventricular cavity. Subsequently, (i) an $18 \mathrm{G}$ needle puncture (or micropuncture) [2] is performed on the membrane of the hemostatic valve of the Impella sheath in its upper left or right quadrant (Figure 1); (ii) a 0.035 " guidewire is then advanced, and a 6 or 7 French sheath is gently introduced for $\mathrm{PCl}$. After $\mathrm{PCl}$, the 6 or 7 French sheath can be removed since the defect closes by itself [1]. If the Impella catheter needs to be removed, it can be done in the standard fashion. If the Impella needs to be maintained for left ventricular support after the procedure, the 6 or 7 French $\mathrm{PCl}$ sheath can be simply removed without damage or bleeding in the homeostatic valve.

\section{Case series}

\section{Case 1}

An 82-year-old man with a previous myocardial infarction and coronary artery bypass grafting was admitted to the emergency department with non-ST-elevation myocardial infarction (non-STEMI (peak troponin $\mathrm{T}$ of 223 pg/ml; normal limit < 14 pg/ml)), cardiogenic shock, and acute renal injury requiring replacement renal therapy (hemodialysis). The echocardiogram showed severe ventricular dysfunction with left ventricle ejection fraction (LVEF) of $25 \%$. Coronary angiography showed the native right coronary artery (RCA) and left anterior descending (LAD) occluded, with both the left main and left circumflex (LCX) arteries showing severe stenosis (Figure 2 A). All saphenous vein grafts were also occluded, and the left internal mammary anastomosis was patent but with distal diffuse disease. Cardiac magnetic resonance imaging showed LVEF of $16 \%$ and viable myocardium in

\section{Corresponding author:}

Adriano Caixeta MD, Department of Interventional Cardiology, Hospital Israelita Albert Einstein, São Paulo, Brazil, phone: 55-11-95236-2000, e-mail: acaixeta@me.com

Received: 10.02.2021, accepted: 8.04.2021. 
the LCX territory only. Left main and LCX high-risk $\mathrm{PCI}$ with Impella support was planned. A 14 French arterial sheath was introduced in the right femoral artery percutaneously. The SHiP technique was performed with puncture of the upper right quadrant membrane (2 o'clock) followed by $\mathrm{PCl}$ with implantation of 3 drug-eluting stents (DES) (Figures 2 B and C). The Impella catheter was removed at the end of the procedure using Perclose ProGlide (Abbott, Chicago, Illinois) sutures without vascular complications or local bleeding.

\section{Case 2}

A 71-year-old man with advanced ischemic cardiomyopathy was hospitalized due to a surgical resection procedure of a scalp tumor; in the postoperative period he developed non-STEMI (peak troponin T of 442 pg/ml; normal limit < $14 \mathrm{pg} / \mathrm{ml}$ ) and refractory cardiogenic shock. The echocardiogram showed LVEF of $28 \%$, and coronary angiography showed in-stent restenosis of the left anterior descending artery occlusion and chronic total occlusion (CTO) in the proximal RCA (Figure $3 \mathrm{~A}$ ). Due to the high surgical/prohibitive risk, LAD PCI with
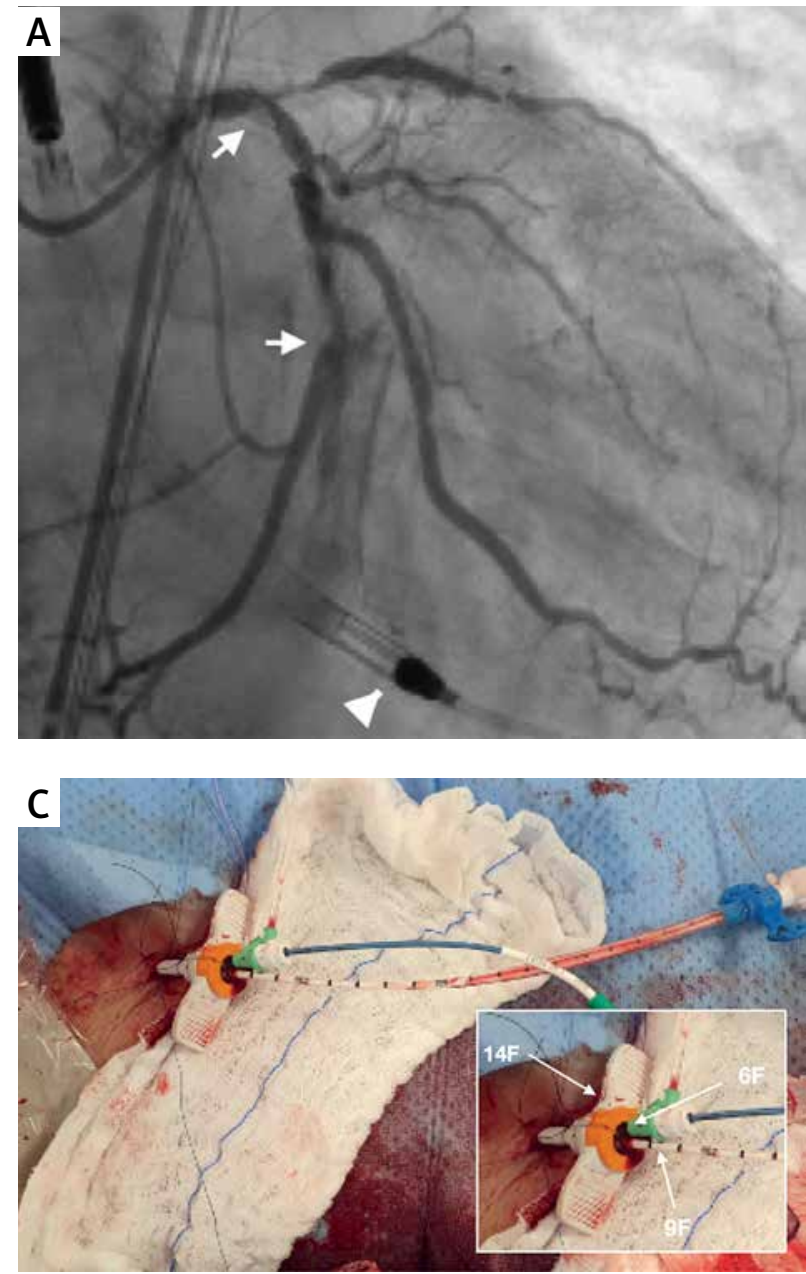

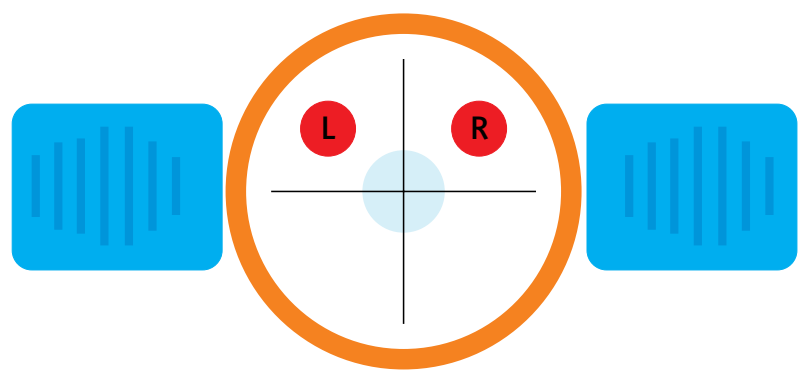

Figure 1. Schematic representation of Impella introducer cross-section. Right (R) or left (L) puncture sites (circles in red) suitable for SHiP. Orange represents the perimeter of the homeostatic membrane. Adapted from Wollmuth et al. [2]

DES implantation was initially performed, followed by a staged RCA CTO $\mathrm{PCl}$ procedure 3 days later utilizing the SHiP technique. A 14 French arterial sheath was also introduced in the right femoral artery percutaneously. A puncture access $18 \mathrm{G}$ needle was used to puncture the hemostasis valve (diaphragm) in the upper right quadrant (2 o'clock) of the sheath, away from the central

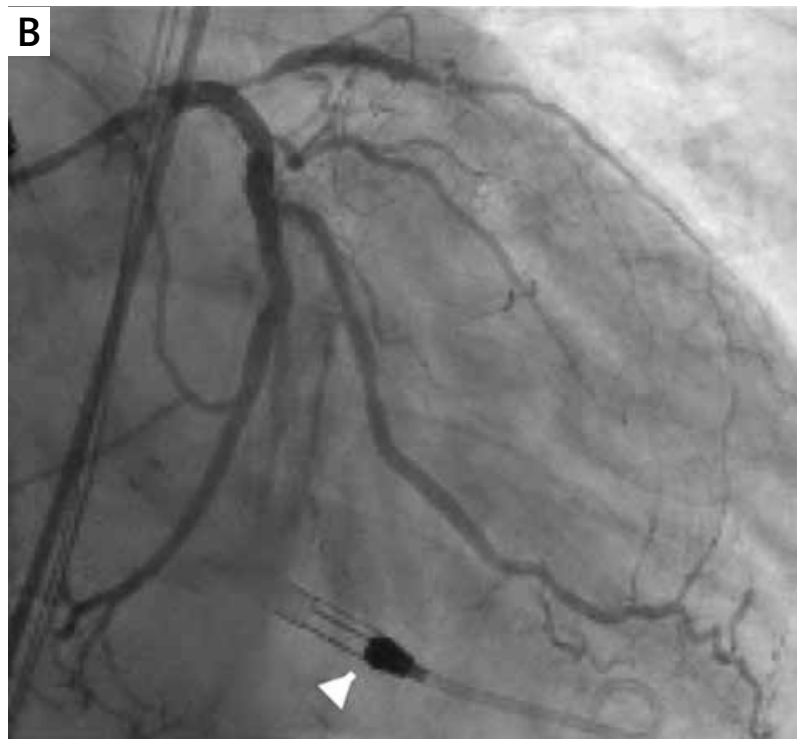

Figure 2. Pre- and post- $\mathrm{PCl}$ coronary angiography (top). Right anterior oblique view showing long and severe lesion in the unprotected left main disease artery and the proximal and mid LCX before ( $A$, arrows) and after (B) the procedure. Note the Impella in the left ventricle ( $A$ and $\mathbf{B}$, head arrow). Vascular access site during the SHiP technique (bottom). The right groin showing 14F Impella sheath (arrow, orange), Impella shaft (white, arrow), and 6F sheath for guidewire $\mathrm{PCl}$ (arrow, green). The 6 French guiding catheter was introduced into the upper right side of the Impella membrane between the 14 French Impella sheath and its 9 French shaft (C) 

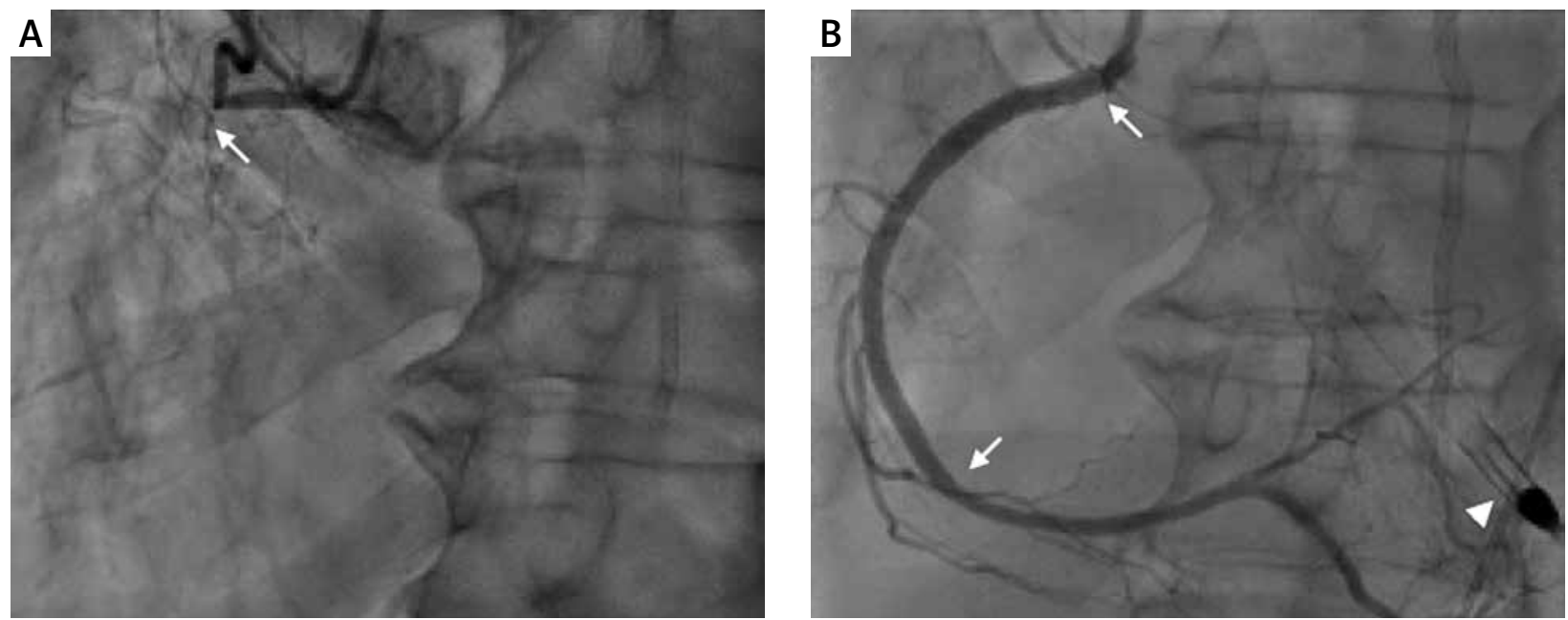

Figure 3. Pre- and post-PCl coronary angiography. RCA CTO before (A) and after (B) DES implantation with Impella support (A and $\mathbf{B}$, head arrow)
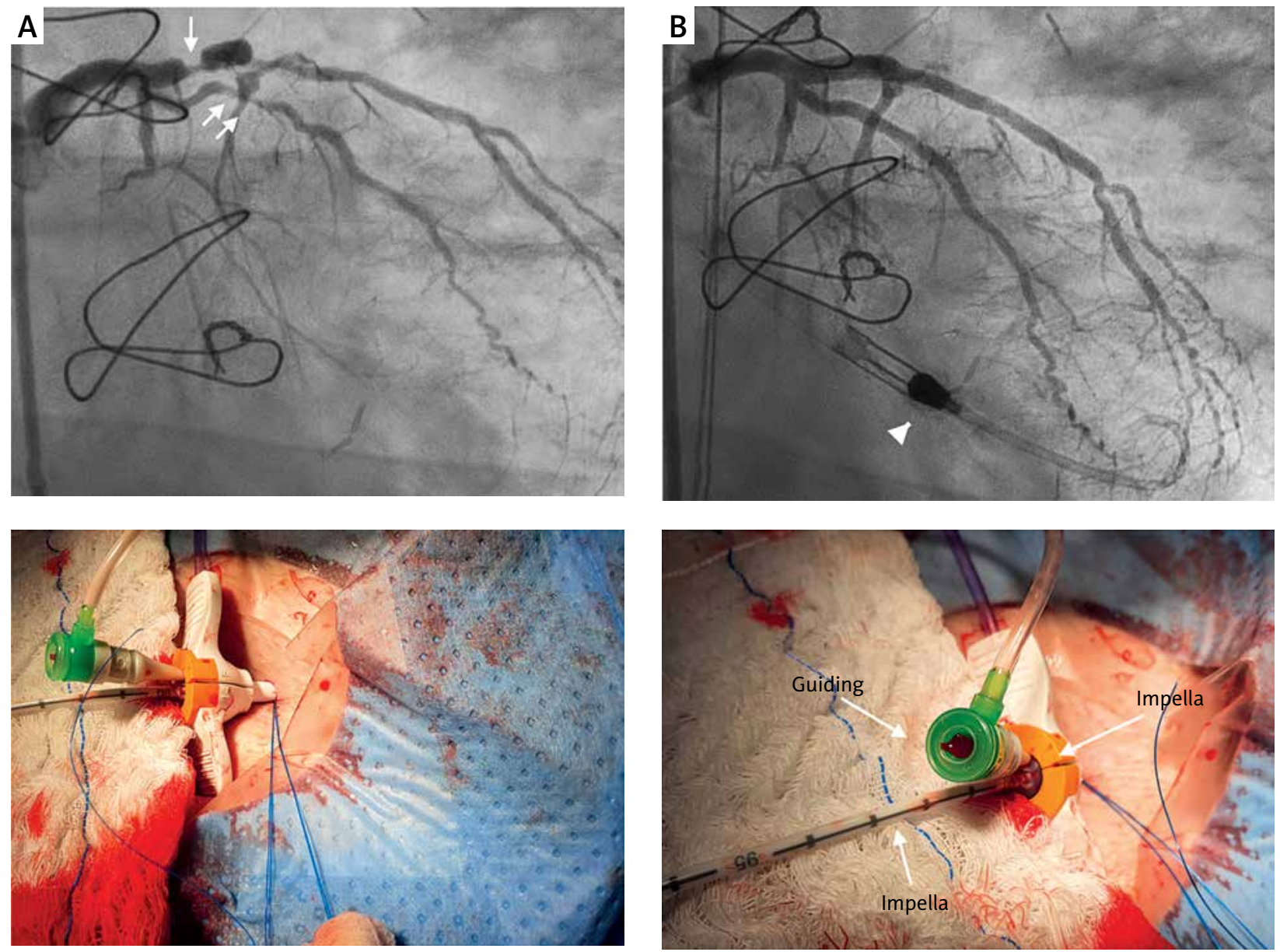

Figure 4. Pre- and post-PCI left coronary angiography (top). Obtuse marginal and major diagonal before (A) and after (B) DES implantation with Impella support ( $\mathbf{A}$ and $\mathbf{B}$, head arrow). Left vascular access for the SHiP technique (bottom). The left groin showing $14 \mathrm{~F}$ Impella sheath (arrow, orange), Impella shaft (white, arrow), and 6F sheath for guidewire $\mathrm{PCl}$ (arrow, green). The 6 French guiding catheter was introduced into the upper left side of the Impella membrane between the 14 French Impella sheath and its 9 French shaft 
lumen, with care to avoid piercing the Impella CP catheter [2-4]. By antegrade wire escalation, СTO PCI was successfully performed with implantation of three DES (Figure $3 \mathrm{~B}$ ). The Impella was left for $24 \mathrm{~h}$ and removed with an arteriotomy hemostasis Perclose ProGlide suture device without complications or bleeding.

\section{Case 3}

A 79-year-old man with diabetes, chronic obstructive pulmonary disease and atrial fibrillation, prior coronary artery bypass graft and heart failure was hospitalized in the emergency department with non-STEMI and cardiogenic shock. High-sensitivity troponin T was 342 pg/ml (normal limit $<14 \mathrm{pg} / \mathrm{ml}$ ). The echocardiogram showed LVEF of $28 \%$, and coronary angiography showed a severe lesion in the first diagonal and first obtuse marginal; all the other native coronary arteries were occluded (Figure $4 \mathrm{~A}$ ). The SHiP technique (Figure 4) was used with a 14 French arterial sheath introduced percutaneously through the left femoral artery. A micropuncture access with a 0.021" needle was used to puncture the hemostasis valve (diaphragm) in the upper left quadrant (10 o'clock) of the sheath. PCI with DES implantation in the diagonal and marginal obtuse branch was successfully performed (Figure 4 B). The Impella was removed at the end of the procedure with hemostasis with one Perclose ProGlide suture device without any complications or bleeding.

\section{Discussion}

The Impella assist device is considered in high-risk $\mathrm{PCl}$, with complex coronary artery disease involving a large territory and severe left ventricular dysfunction [5]. Kumar et al. [1] were the first to describe the technique in 2019, in which successful multivessel PCI was performed via an Impella CP sheath [2].The Impella CP peel-away introducer sheath and Impella motor are 14 French in diameter, but the catheter shaft between the motor housing and the external red Impella plug is 9 French in diameter. Therefore, the Impella CP introducer sheath has been used to introduce guide catheters and perform angiography and $\mathrm{PCl}$ while simultaneously using the Impella CP for hemodynamic support [1, 2].

This novel technique has been modified successfully by Dr. Jason Wollmuth [6], where the diaphragm of the Impella sheath is punctured, and a 6 or 7 French sheath can be introduced through which angiography and $\mathrm{PCl}$ can be performed. It is important to identify the correct site of the $14 \mathrm{~F}$ introducer membrane puncture (upper quadrants) so as to avoid the center of the membrane where the Impella body (shaft) catheter is located. Conversely, the simple insertion of the 6 or 7 French $\mathrm{PCl}$ introducer into the same orifice through which the Impella catheter is inserted - as in the case of Kumar et al. [1] without puncturing the membrane at the recommended site may modify its geometry, thereby preventing homeostasis and potentially leading to continuous bleeding [2]. Thus, $\mathrm{PCl}$ by the SHiP technique can be performed with a 6 French or 7 French [1-4] introducer, short or long, with (preferably) or without a hydrophilic coating.

In 2020, Wollmuth et al. [2] published a series of 17 cases that showed that with the SHiP technique it was possible to perform concomitant rotational atherectomy (88\%), multivessel PCI (94\%), and chronic total occlusion treatment (34\%) in a safe manner despite the prolonged duration of these procedures. In all cases, no thrombus formation was noted in the devices after removal of the Impella introducer and catheter except in 1 case in which thrombus was reported in the iliac artery 7 days after its implantation. Similarly, in our 3 high-risk $\mathrm{PCl}$ cases the procedures were performed safely and without vascular complications or major bleeding. Unlike Wollmuth et al. [2], who used a 7 French $\times 45 \mathrm{~cm}$ sheath with a $35 \mathrm{~cm}$ hydrophilic coating in $\sim 80 \%$ of the cases, in our cases, $\mathrm{PCls}$ were performed with a non-hydrophilic 6 French $\times 11 \mathrm{~cm}$ sheath in 2 cases (Avanti, Cordis, Santa Clara, California), and in the last case, a hydrophilic 6 French $\times 23 \mathrm{~cm}$ sheath was used (Radiofocus, Terumo, Japan).

Potential limitations of the SHiP technique include (i) bleeding around the sheath, (ii) bleeding through the diaphragm, (iii) disruption of the integrity of the Impella CP peel-away introducer sheath, (iv) damage to the Impella CP catheter while taking the second access, (v) interaction between guiding catheter and Impella CP catheter with possible displacement, (vi) difficulties in guiding catheter engagement and manipulation, (vii) lack of second access to deal with ipsilateral vascular access complications, and finally (viii) manipulation of the guide catheter during PCI can cause bleeding around the $14 \mathrm{~F}$ sheath and, consequently, problems with hemostasis [2, 4].

Our 3 cases add additional information to the 19 cases already published in the literature along with several cases in social media [7] on the feasibility and safety of the SHiP technique. The SHiP technique facilitates complex $\mathrm{PCl}$ in subgroups of high-risk patients by determining rapid and practical vascular access and avoiding the need for puncture, manipulation, and homeostasis of a second arterial access site. Hence, the use of this new technique has the potential to minimize vascular complications and bleeding. Despite its initial experience, the $\mathrm{SHiP}$ technique may become a default procedure in the treatment of high-risk $\mathrm{PCl}$ patients who require concomitant Impella implantation.

\section{Conclusions}

The use of the SHiP technique is simple, safe, and reproducible, making it possible to perform percutaneous coronary revascularization procedures and mechanical circulatory support with the Impella using single arterial access. 


\section{Conflict of interest}

The authors declare no conflict of interest.

\section{References}

1. Kumar K, Reddy S, Acharya D, Lotun K. Novel technique of performing multivessel PCI through an Impella sheath. Catheter Cardiovasc Interv 2020; 96: 117-20.

2. Wollmuth J, Korngold E, Croce, K, Pinto DS. The Single-access for Hi-risk PCI (SHiP) technique. Catheter Cardiovasc Interv 2020; 96: 114-6.

3. Nagaraja V, Khatri JJ. Hybrid robotic Impella assisted single arterial access complex high risk percutaneous coronary intervention. Cardiovasc Revasc Med 2020; 21: 105-7.

4. Ahmed T, Lodhi SH, Ahmed T, et al. Fill two needs with one deed! Single-access protected percutaneous coronary intervention with Impella hemodynamic support. CJC Open 2020; 2: 77-8.

5. Rihal CS, Naidu SS, Givertz MM, et al.; Society for Cardiovascular Angiography and Interventions (SCAl); Heart Failure Society of America (HFSA); Society of Thoracic Surgeons (STS); American Heart Association (AHA), and American College of Cardiology (ACC). 2015 SCAI/ACC/HFSA/STS clinical expert consensus statement on the use of percutaneous mechanical circulatory support devices in cardiovascular care: endorsed by the American Heart Association, the Cardiological Society of India, and Sociedad Latino Americana de Cardiologia Intervencion; Affirmation of Value by the Canadian Association of Interventional Cardiology-Association Canadienne de Cardiologie d'intervention. J Am Coll Cardiol 2015; 65: e7-26.

6. https://twitter.com/jason_wollmuth/status/ 1179138613050667013 ?s=09/citations-a-twitter. Accessed 18 March 2021.

7. Kaplan AV, Wolmuth J, Pinto DS. Impella supported PCI via a single access sheath - Introduction and rapid adoption: the Power of Social Media. Eurolntervention 2020 - Images in Cardiology. Available in https://www.pcronline.com/Cases-resources-images/Images-interventional-cardiology/Eurolntervention-images/ IMPELLA-supported-PCl-single-access-sheath-introduction-rapid-adoption. Accessed 18 $8^{\text {th }}$ March 2021. 